\title{
Parsing with Probabilistic Strictly Locally Testable Tree Languages
}

\author{
Jose Luis Verdú-Mas, Rafael C. Carrasco, and Jorge Calera-Rubio
}

\begin{abstract}
Probabilistic $k$-testable models (usually known as $k$-gram models in the case of strings) can be easily identified from samples and allow for smoothing techniques to deal with unseen events during pattern classification. In this paper, we introduce the family of stochastic $k$-testable tree languages and describe how these models can approximate any stochastic rational tree language. The model is applied to the task of learning a probabilistic $k$-testable model from a sample of parsed sentences. In particular, a parser for a natural language grammar that incorporates smoothing is shown.
\end{abstract}

Index Terms-Parsing with probabilistic grammars, stochastic learning, tree grammars.

\section{INTRODUCTION}

STOchastic models based on $k$-grams predict the probSability of the next symbol in a sequence as a function of the $k-1$ previous symbols and have been widely used in natural language processing [1], [2], [3]. From a theoretical (albeit not historical) point of view, $k$-gram models can be regarded as a probabilistic extension of strictly locally testable string languages [4]. Strictly locally testable models or $k$-testable models, ${ }^{1}$ a kind of model that classifies strings by looking at substrings of length at most $k$, are easy to learn from samples and can be dealt with using efficient and simple algorithms [5], [6], [7]. It is also straightforward to incorporate probabilities into the model, although data sparseness often leads to the assignment of null probabilities to many strings. To alleviate this, there is a number of well-known techniques [8], [9] to smooth the distribution.

When hierarchical relations are established among the data components, trees become a more adequate representation than strings of symbols. This is the case, for example, in natural language parsing [10], [11], [12], [13] or structured text processing [14], [15]. Context-free grammars [16] provide a traditional formalism that handles structural information. This kind of grammars can be easily written and updated by humans, although it is difficult to learn them automatically. For instance, it is hard to find the appropriate degree of generalization unless some information about the size of the target grammar is available [17].

The class of parse trees generated by a context-free grammar can be characterized as a rational tree language [18], [19] and any rational tree language can be recognized by a bottom-up (also called frontier-to-root or ascending)

1. In their original denomination [4], these models were called $k$-testable in the strict sense but, in the following, we will simply refer to them as $k$-testable.

- The authors are with the Departament de Llenguatges $i$ Sistemes Informatics, Universidad de Alicante, E-03071 Alicante, Spain.

E-mail: \{verdu, calera, carrasco\}@dlsi.ua.es.

Manuscript received 5 Jan. 2004; revised 2 Aug. 2004; accepted 20 Sept. 2004; published online 12 May 2005.

Recommended for acceptance by M. Basu.

For information on obtaining reprints of this article, please send e-mail to: tpami@computer.organd reference IEEECSLog NumberTPAMISI-0011-0104. finite-state tree automaton. ${ }^{2}$ The class of $k$-testable tree languages [21] is a proper subclass of the class of rational tree languages, where the effect of events that have occurred beyond a certain depth window are ignored when processing a tree. Then, $k$-testable tree models can be regarded as a special case of automata whose states are simply subtrees truncated to a certain depth. If valence (that is, number of children) remains bounded, the number of states in the automaton remains finite (and observable).

Stochastic models that assign a probability to a tree can be useful to select the best parse tree for a sentence and resolve structural ambiguity. For instance, in many natural-language processing applications, obtaining the correct syntactical structure for a sentence is an important intermediate step before assigning an interpretation to it. But, ambiguous parses are very common in real natural-language sentences (e.g., those longer than 15 words; the fact that many ambiguous parse examples in books sound a bit awkward is due to the fact that they involve short sentences [22], [23, p. 411]). Choosing the correct parse for a given sentence is a crucial task if one wants to interpret the meaning of the sentence, due to the principle of compositionality [24, p. 358], which states, informally, that the interpretation of a sentence is obtained by composing the meanings of its constituents according to the groupings defined by the parse tree. ${ }^{3}$

As will be introduced in this paper, a class of probabilistic models analogous to the $k$-gram models can be defined for tree languages. In this probabilistic extension, the automaton behaves as a top-down generating device, but the computation is still performed bottom-up: The state that generates a subtree can be recognized by a finite-state computation over the generated subtree or, more precisely, over those nodes lying within a depth $k$ in the subtree.

Our notation is introduced in Section 2 and some known properties of strictly locally testable tree languages are presented in Section 3. In Section 4, we show how to obtain

2. It is known [18], [20] that the class of languages that can be recognized by deterministic top-down (root-to-frontier or descending) tree automata is a proper subset of those recognizable by bottom-up automata.

3. The principle of compositionality in natural language is akin to the concept of syntax-directed translation used in compiler construction [25, p. 25] and to the principles which inspire the syntactic transfer architecture used in machine translation systems [26]. 


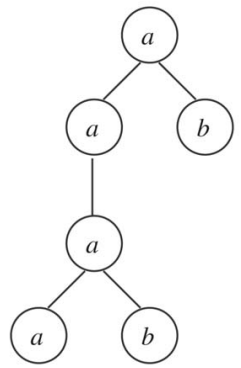

Fig. 1. A graphical representation of the tree $a(a(a(a b)) b)$.

from a given, general, tree automaton the $k$-testable automaton that minimizes the relative entropy or KullbackLeibler divergence [27] to the original one. In Section 5, we justify how the best model from a stochastic sample is obtained. The application of these models to describe the syntactical structure of sentences is presented in Section 6, together with some techniques to smooth the distributions. Experiments using one of these models, called child-annotated model for short, for structural disambiguation are presented in Section 7 and compared with other family-annotated models. Finally, conclusions are given in Section 8.

\section{Trees and Tree Automata}

Given a finite set of symbols (called the alphabet) $\Sigma=\left\{\sigma_{1}, \ldots, \sigma_{|\Sigma|}\right\}$, the language $T_{\Sigma}$ of $\Sigma$-trees can be defined recursively. On the one hand, all symbols in $\Sigma$ are $\Sigma$-trees whose depth is zero. On the other hand, given a symbol $\sigma \in \Sigma$ and $m>0 \Sigma$-trees $t_{1}, \ldots, t_{m}, \sigma\left(t_{1} \cdots t_{m}\right)$ is a new $\Sigma$-tree in $T_{\Sigma}$ of depth $1+\max _{i}\left\{\operatorname{depth}\left(t_{i}\right)\right\}$ and with root label $\sigma$. For instance, the depth $3\{a, b\}$-tree $a(a(a(a b)) b)$ in $T_{\{a, b\}}$ is depicted in Fig. 1. In the following, we will assume that $\Sigma$ is given and then, we will simply use the name trees for all $t \in T_{\Sigma}$ and, labels for all $\sigma \in \Sigma$. Any subset of $T_{\Sigma}$ will be called a tree language. The set of subtrees of a tree $t$ is

$$
\operatorname{sub}(t)= \begin{cases}\{\sigma\} & \text { if } t=\sigma \in \Sigma \\ \{t\} \cup \bigcup_{i=1}^{m} \operatorname{sub}\left(t_{i}\right) & \text { if } t=\sigma\left(t_{1} \cdots t_{m}\right) \in T_{\Sigma}-\Sigma .\end{cases}
$$

Finite-state automata are processing devices with a finite number of states. In contrast to the case of strings, where the automaton computes a new state for every prefix [16], a frontier-to-root tree automaton processes the tree in a bottomup fashion and a state is computed for every subtree. If the subtree consists of a root node with $m$ subtrees, the state is computed as a function of the root label and of the $m$ states obtained after processing the subtrees. Therefore, the automaton needs a collection of transition functions, one for each possible value of $m$.

Formally, a deterministic finite-state tree automaton (DTA) is defined as $A=(Q, \Sigma, \Delta, F)$, where $Q=$ $\left\{q_{1}, \ldots, q_{|Q|}\right\}$ is a finite set of states, $\Sigma=\left\{\sigma_{1}, \ldots, \sigma_{|\Sigma|}\right\}$ is the alphabet, $F \subseteq Q$ is the subset of accepting states, and $\Delta=\left\{\delta_{0}, \delta_{1}, \ldots, \delta_{M}\right\}$ is a collection of transition functions of the form $\delta_{m}: \Sigma \times Q^{m} \rightarrow Q$. For every tree $t \in T_{\Sigma}$, the output $\delta(t) \in Q$ when $A$ operates on $t$ is

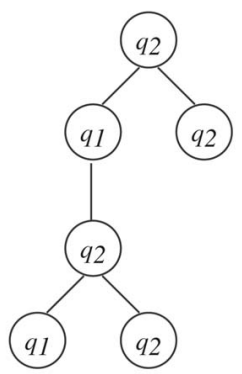

Fig. 2. Output states after the computation of the tree automaton $A$ on $a(a(a(a b)) b)$.

$$
\delta(t)= \begin{cases}\delta_{0}(\sigma) & \text { if } t=\sigma \in \Sigma \\ \delta_{m}\left(\sigma, \delta\left(t_{1}\right), \ldots, \delta\left(t_{m}\right)\right) & \text { if } t=\sigma\left(t_{1} \cdots t_{m}\right) \in T_{\Sigma}-\Sigma\end{cases}
$$

For instance, if $\delta_{0}(a)=q_{1}, \delta_{0}(b)=q_{2}, \delta_{2}\left(a, q_{1}, q_{2}\right)=q_{2}$, and $\delta_{1}\left(a, q_{2}\right)=q_{1}$, the result of the operation of $A$ on tree $a(a(a(a b)) b)$ depicted in Fig. 1 is $\delta(t)=\delta_{2}(a, \delta(a(a(a b)))$, $\delta(b))$. Recursively, one gets $\delta(t)=\delta_{2}\left(a, q_{1}, q_{2}\right)=q_{2}$, as shown in Fig. 2.

The tree language $L(q)$ accepted by state $q \in Q$ is the subset of trees in $T_{\Sigma}$ with output $q$

$$
L(q)=\left\{t \in T_{\Sigma}: \delta(t)=q\right\}
$$

and the tree language $L(A)$ recognized by the automaton $A$ is the subset of trees in $T_{\Sigma}$ whose output is a state in $F$

$$
L(A)=\bigcup_{q \in F} L(q) .
$$

Each language that can be recognized by a DTA is a rational tree language. In case that a transition function $\delta_{m}$ is a partial mapping, all undefined transitions are assumed to lead to a special absorption state $\perp \notin F$. With this assumption, the languages $L(q)$ define a partition of $T_{\Sigma}$.

Probabilistic tree automata generate a probability distribution over the trees in $T_{\Sigma}$ by using only a finite number of parameters and are more naturally seen as top-down generating devices. In particular, a probabilistic DTA incorporates a probability for every transition in the automaton, normalized so that the probabilities of the transitions leading to the same state add up to one. In other words, there is a collection of functions $P=\left\{p_{0}, p_{1}, p_{2}, \ldots, p_{M}\right\}$ of the type $p_{m}: \Sigma \times Q^{m} \rightarrow[0,1]$ such that they satisfy, for all $q \in Q$,

$$
\sum_{\sigma \in \Sigma} \sum_{m=0}^{M} \sum_{\substack{i_{1}, \ldots, i_{m} \in Q: \\ \delta_{m}\left(\sigma, i_{1}, \ldots, i_{m}\right)=q}} p_{m}\left(\sigma, i_{1}, \ldots, i_{m}\right)=1 .
$$

With this normalization, $P$ defines a probability distribution $^{4}$ over every $L(q)$. In order to define a probability distribution over $T_{\Sigma}$, every probabilistic deterministic tree automaton (PDTA) $A=(Q, \Sigma, \delta, P, \rho)$ also provides a function $\rho: Q \rightarrow[0,1]$ which, for every $q \in Q$, gives the probability of the class $L(q)$ and satisfies

$$
\sum_{q \in Q} \rho(q)=1 .
$$

4. Although additional constraints are necessary to guarantee its consistency, see [28]. 


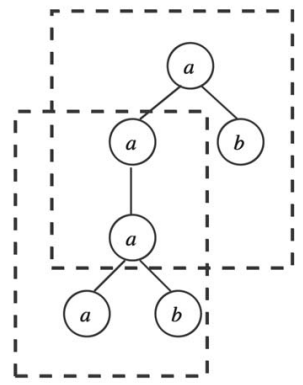

(a)

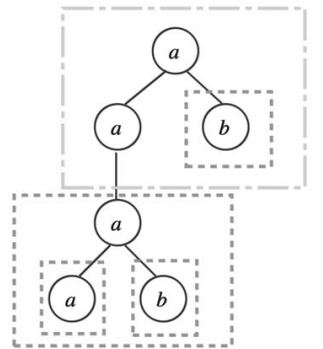

(b)
Fig. 3. (a) Set of 3-forks contained in $a(a(a(a b)) b)$. (b) Its 2-root (dashdotted) and 2-subtrees.

Then, the probability of a tree $t$ in the stochastic language generated by $A$ is defined as

$$
p(t \mid A)=\rho(\delta(t)) \pi(t),
$$

where $\pi(t)$ is the product of the probabilities of all the transitions performed when $A$ operates on $t$, that is,

$$
\begin{aligned}
& \pi(t)= \\
& \left\{\begin{array}{cc}
p_{0}(\sigma) & \text { if } t=\sigma \in \Sigma \\
p_{m}\left(\sigma, \delta\left(t_{1}\right), \ldots, \delta\left(t_{m}\right)\right) & \\
\pi\left(t_{1}\right) \cdots \pi\left(t_{m}\right) & \text { if } t=\sigma\left(t_{1} \cdots t_{m}\right) \in T_{\Sigma}-\Sigma .
\end{array}\right.
\end{aligned}
$$

For instance, in the example used to draw Fig. 2, the probability of tree $a(a(a(a b)) b)$ computed by the PDTA is $\rho\left(q_{2}\right) p_{2}\left(a, q_{1}, q_{2}\right)^{2} p_{1}\left(a, q_{2}\right) p_{0}(b)^{2} p_{0}(a)$. Any probability distribution over $T_{\Sigma}$ defined by a PDTA will be called a stochastic rational tree language.

\section{Strictly Locally Testable Tree LANGUAGES}

Strictly locally testable languages are characterized, in the case of strings, by defining

1. the allowed set of substrings of length $k$ and

2. the sets of allowed prefixes and suffixes of length strictly smaller than $k$.

In the case of trees, as described by Knuutila [21], the concept of $k$-fork plays the role of the substrings and the $k$-root and $k$-subtrees play the role of the prefixes and suffixes. Every $k$-fork contains a node and all its descendents lying at a depth smaller that $k$. The $k$-root of a tree is its topmost $k$-fork and the $k$-subtrees are all the subtrees whose depth is smaller than $k$. These concepts are illustrated in Fig. 3 and formally defined below.

For all $k>0$ and for all trees $t=\sigma\left(t_{1} \cdots t_{m}\right) \in T_{\Sigma}$, the $k$-root of $t$ is the tree in $T_{\Sigma}$ is defined as

$$
r_{k}\left(\sigma\left(t_{1} \cdots t_{m}\right)\right)= \begin{cases}\sigma & \text { if } k=1 \\ \sigma\left(r_{k-1}\left(t_{1}\right) \cdots r_{k-1}\left(t_{m}\right)\right) & \text { otherwise. }\end{cases}
$$

In case $m=0$, that, is $t=\sigma \in \Sigma$, then $r_{k}(\sigma)=\sigma$.

On the other hand, the set $f_{k}(t)$ of $k$-forks and the set $s_{k}(t)$ of $k$-subtrees of a tree $t$ are defined for all $k>0$ as follows:

$$
\begin{aligned}
& f_{k}\left(\sigma\left(t_{1} \cdots t_{m}\right)\right)= \\
& \begin{cases}\emptyset & \text { if } \operatorname{depth}\left(\sigma\left(t_{1} \cdots t_{m}\right)\right)<k-1 \\
\left\{r_{k}\left(\sigma\left(t_{1} \cdots t_{m}\right)\right)\right\} \cup \bigcup_{i=1}^{m} f_{k}\left(t_{i}\right) & \text { otherwise. }\end{cases}
\end{aligned}
$$

$$
\begin{aligned}
& s_{k}\left(\sigma\left(t_{1} \cdots t_{m}\right)\right)= \\
& \begin{cases}\sigma\left(t_{1} \cdots t_{m}\right) \cup \bigcup_{i=1}^{m} s_{k}\left(t_{i}\right) & \text { if } \operatorname{depth}\left(\sigma\left(t_{1} \cdots t_{m}\right)\right)<k \\
\bigcup_{i=1}^{m} s_{k}\left(t_{i}\right) & \text { otherwise. }\end{cases}
\end{aligned}
$$

In the particular case $t=\sigma \in \Sigma$, then $s_{k}(t)=f_{1}(t)=\sigma$ and $f_{k}(t)=\emptyset$ for all $k>1$. For instance, if $t=a(a(a(a b)) b)$, then one gets $r_{2}(t)=\{a(a b)\}, f_{3}(t)=\{a(a(a) b), a(a(a(b)))\}$, and $s_{2}(t)=\{a(a b), a, b\}$, as shown in Fig. 3 .

A $k$-testable tree language consists of trees whose $k$-forks, $(k-1)$-subtrees, and $(k-1)$-root belong to predefined sets. More precisely, a tree language $T \subseteq T_{\Sigma}$ is $k$-testable (with $k \geq 2$ ) if there exist three finite subsets $\mathcal{R}, \mathcal{F}, \mathcal{S} \subseteq T_{\Sigma}$ such that

$$
t \in T \Leftrightarrow r_{k-1}(t) \in \mathcal{R} \wedge f_{k}(t) \subseteq \mathcal{F} \wedge s_{k-1}(t) \subseteq \mathcal{S} .
$$

Without loss of generality, it can be assumed that $\mathcal{R} \subseteq$ $r_{k-1}(\mathcal{F} \cup \mathcal{S})$ and $\mathcal{S} \subseteq s_{k-1}(\mathcal{F})$. With these assumptions, it can be proven that any $k$-testable language is rational [21]. Indeed, the DTA $(Q, \Sigma, \Delta, F)$ with

$$
\begin{aligned}
Q & =r_{k-1}(\mathcal{F} \cup \mathcal{S}) \\
F & =\mathcal{R}
\end{aligned}
$$

$\delta_{m}\left(\sigma, t_{1}, \ldots, t_{m}\right)= \begin{cases}r_{k-1}\left(\sigma\left(t_{1} \cdots t_{m}\right)\right) & \text { if } \sigma\left(t_{1} \cdots t_{m}\right) \in \mathcal{F} \cup \mathcal{S} \\ \perp & \text { otherwise }\end{cases}$

recognizes the language $T$ defined by (12). We will call the automaton obtained in this way a $k$-testable DTA.

As proven by Knuutila [21], the $k$-testable class can be identified in the limit [29] from positive samples and, given a sample set $\Omega=\left\{\tau_{1}, \tau_{2}, \tau_{3}, \ldots, \tau_{|\Omega|}\right\}$ of trees in the language $T$, using $\mathcal{R}=r_{k-1}(\Omega), \mathcal{F}=f_{k}(\Omega)$, and $\mathcal{S}=s_{k-1}(\Omega)$, defines the minimal $k$-testable model that recognizes $\Omega$. As an illustration, consider the single-example sample $\Omega=\{a(a(a(a b)) b)\}$ and $k=3$. Then, one gets the set of states $Q=\{a(a b)$, $a(a), a, b\}$ with acceptance subset $F=\{a(a b)\}$. The defined transitions are $\delta_{0}(a)=a, \delta_{0}(b)=b, \delta_{2}(a, a, b)=a(a b), \delta_{2}(a$, $a(a), b)=a(a b)$, and $\delta_{1}(a, a(a b))=a(a)$.

In the following, we will call a probabilistic $k$-testable tree automaton any PDTA whose structure (that is, the transitions and states) is built according to (13) and will call the distribution generated by a PDTA of this type a stochastic $k$-testable tree language. Therefore, stochastic $k$-testable tree languages are a proper subclass of stochastic rational tree languages. However, as will be shown in the next section, a $k$-testable model that approximates a given stochastic rational tree language can be efficiently obtained.

\section{Approximating Probabilistic DTA by $k$-Testable Automata}

This section describes a procedure to build a probabilistic $k$-testable tree automaton upon a given DTA so that the relative entropy [27] to a second probabilistic automaton is minimal. In the case of strings, Stolcke and Segal [30] describe 
a procedure to obtain a $k$-gram model from a probabilistic context-free grammar, although its relation to the relative entropy is not shown. Here, we will start by defining the states and transitions of the $k$-testable tree automaton and, then, we will describe how the probabilities are computed.

\subsection{States and Transitions}

Assume that we are given a general probabilistic DTA $A=$ $(Q, \Sigma, \Delta, P, \rho)$ and, for a certain $k>1$, we want to obtain a probabilistic $k$-testable tree automaton $A^{\prime}=\left(Q^{\prime}, \Sigma, \Delta^{\prime}, P^{\prime}, \rho^{\prime}\right)$ such that the relative entropy (see Section 4.2) between $A$ and $A^{\prime}$ is minimal. In order to find the states and transitions in $A^{\prime}$, one needs to know the set $\Theta^{[k]}$ of $k$-forks and $(k-1)$-subtrees that $A$ may generate, that is,

$$
\Theta^{[k]}=\left\{r_{k}(t): t \in T_{\Sigma} \wedge \delta(t) \neq \perp\right\}=\bigcup_{q \neq \perp} r_{k}(L(q)) .
$$

The sets $r_{k}(L(q))$ can be efficiently built by means of an iterative procedure:

$$
\begin{aligned}
r_{1}(L(q))= & \left\{\sigma \in \Sigma: \delta_{0}(\sigma)=q\right\} \cup \\
& \left\{\sigma \in \Sigma: \exists i_{1}, \ldots, i_{m} \in Q: \delta_{m}\left(\sigma, i_{1}, \ldots, i_{m}\right)=q\right\} .
\end{aligned}
$$

And, for all $k>1$,

$$
\begin{aligned}
r_{k}(L(q))= & \left\{\sigma \in \Sigma: \delta_{0}(\sigma)=q\right\} \cup\left\{\sigma\left(t_{1} \cdots t_{m}\right):\right. \\
& \exists i_{1}, \ldots, i_{m} \in Q: \delta_{m}\left(\sigma, i_{1}, \ldots, i_{m}\right)=q \bigwedge \\
& \left.\forall n\left[t_{n} \in r_{k-1}\left(L\left(i_{n}\right)\right)\right]\right\} .
\end{aligned}
$$

From (13), the set of states $Q^{\prime}$ is simply given by $Q^{\prime}=r_{k-1}\left(\Theta^{[k]}\right)=\Theta^{[k-1]}$ and $\Delta^{\prime}$ contains $\delta_{m}^{\prime}\left(\sigma, t_{1}, \ldots, t_{m}\right)=$ $r_{k-1}\left(\sigma\left(t_{1} \cdots t_{m}\right)\right)$ if $\sigma\left(t_{1} \cdots t_{m}\right) \in \Theta^{[k]}$ (and equals $\perp$ otherwise). For instance, let $\Delta$ in the automaton $A$ contain the following transitions:

$$
\begin{aligned}
& \delta_{0}(a)=\delta_{2}\left(a, q_{1}, q_{2}\right)=q_{1}, \\
& \delta_{0}(b)=\delta_{2}\left(a, q_{1}, q_{1}\right)=q_{2},
\end{aligned}
$$

with the rest being undefined. In such a case, $A$ is not $k$-testable and $\Theta^{[1]}=\{a, b\}, Q^{\prime}=\Theta^{[2]}=\{a, b, a(a a), a(a b)\}$ and $\Theta^{[3]}$ contains the 14 trees depicted in Fig. 4. Each tree in $\Theta^{[3]}$ corresponds to a defined transition in $\Delta^{\prime}$ : For instance, the first tree in the third line in the figure corresponds to $\delta_{2}(a, a(a b), b)=a(a b)$.

\subsection{Probabilities}

Next, we proceed to compute the probabilities in $P^{\prime}$ and $\rho^{\prime}$ that minimize the relative entropy. Indeed, the procedure will also be valid to obtain an approximate PDTA with the structure of any given DTA. Recall that the relative entropy $H\left(A, A^{\prime}\right)$ between two PDTA $A$ and $A^{\prime}$ is $H\left(A, A^{\prime}\right)=G\left(A, A^{\prime}\right)-$ $G(A, A)$ with

$$
G\left(A, A^{\prime}\right)=-\sum_{t \in T_{\Sigma}} p(t \mid A) \log p\left(t \mid A^{\prime}\right) .
$$

The component that depends on the model $A^{\prime}$, that is, $G\left(A, A^{\prime}\right)$ can be computed as the addition of two terms [31]:

$$
G_{\rho}\left(A, A^{\prime}\right)=-\sum_{\substack{i \in Q \\ j \in Q^{\prime}}} \eta_{i j} \rho(i) \log \rho^{\prime}(j)
$$
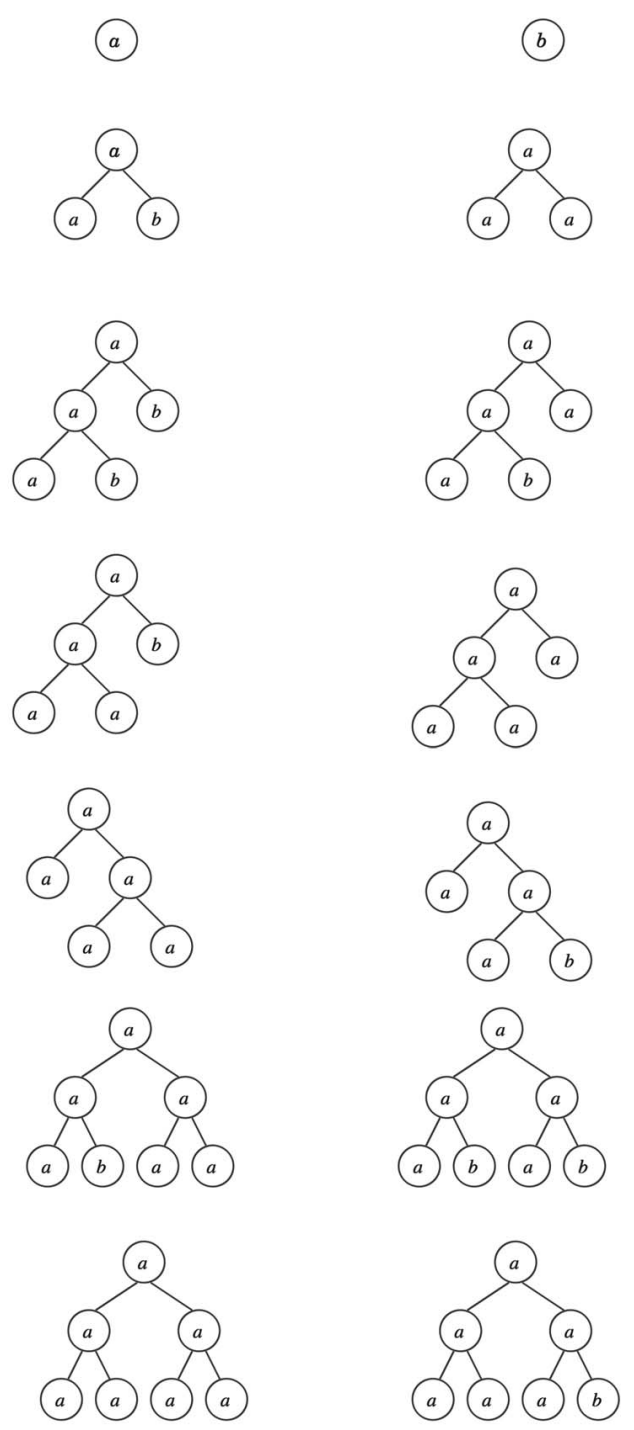

Fig. 4. Set $\Theta^{[3]}$ of 2 -subtrees and 3-forks generated by the automaton $A$ with the transitions defined in (16).

and

$$
\begin{aligned}
G_{\pi}\left(A, A^{\prime}\right)= & \sum_{m=0}^{M} \sum_{\sigma \in \Sigma} \sum_{i_{1}, i_{2}, \ldots, i_{m} \in Q} \sum_{j_{1}, j_{2}, \ldots, j_{m} \in Q^{\prime}} \\
& C_{\delta_{m}\left(\sigma, i_{1}, i_{2}, \ldots, i_{m}\right)} p_{m}\left(\sigma, i_{1}, i_{2}, \ldots, i_{m}\right) \\
& \log p_{m}^{\prime}\left(\sigma, j_{1}, j_{2}, \ldots, j_{m}\right) \eta_{i_{1} j_{1}} \eta_{i_{2} j_{2}} \ldots \eta_{i_{m} j_{m}},
\end{aligned}
$$

where $C_{q}$ is the expected number, according to the automaton $A$, of nodes of type $q$ in a tree and $\eta_{i j}$ represents the probability with respect to $A$ that a node of type $i \in Q$ expands as a subtree $t$ such that $\delta^{\prime}(t)=j$. All the coefficients $C_{q}$ and $\eta_{i j}$ can be efficiently computed using iterative procedures as described in [31]:

$$
\eta_{i j}=\sum_{m=0}^{M} \sum_{\substack{i_{1}, \ldots, i_{m} \in Q: \\ \delta_{m}\left(\sigma, i_{1}, \ldots, i_{m}\right)=i}} \sum_{\substack{j_{1}, \ldots, j_{m} \in Q^{\prime}: \\ \delta_{m}^{\prime}\left(\sigma, j_{1}, \ldots, j_{m}\right)=j}} p_{m}\left(\sigma, i_{1}, \ldots, i_{m}\right) \eta_{i_{1} j_{1}} \eta_{i_{2} j_{2}} \ldots \eta_{i_{m} j_{m}}
$$


TABLE 1

Probabilities $\rho^{\prime}$ for the Approximate 3-Testable Automaton $A^{\prime}$

$$
\begin{array}{ll}
\rho^{\prime}(a)=0.75 & \rho^{\prime}(b)=0 \\
\rho^{\prime}(a(a b))=0.1875 & \rho^{\prime}(a(a a))=0.0625
\end{array}
$$

Note that the possible arguments of $\rho^{\prime}$ are the 2-roots of the trees in Fig. 4.

$$
\begin{aligned}
C_{i}= & \rho(i) \\
& +\sum_{j \in Q} C_{j} \sum_{m=1}^{M} \sum_{\sigma \in \Sigma} \sum_{\substack{j_{1}, j_{2}, \ldots, j_{m} \in Q: \\
\delta\left(\sigma, j_{1}, \ldots, j_{m}\right)=j}} p_{m}\left(\sigma, j_{1}, j_{2}, \ldots, j_{m}\right) \sum_{n=1}^{m} D\left(i, j_{n}\right),
\end{aligned}
$$

where $D(i, j)=1$ if $i=j$ and zero otherwise.

In order to minimize $G_{\rho}$ one has to take into account that $\rho^{\prime}$ is normalized according to (6). Then, we will add a Lagrange multiplier $\mu$ when differentiating with respect to $\rho^{\prime}(j)$ :

$$
\frac{\partial G_{\rho}}{\partial \rho^{\prime}(j)}+\mu=0 .
$$

Then, one gets

$$
\rho^{\prime}(j)=\frac{1}{\mu} \sum_{i \in Q} \eta_{i j} \rho(i),
$$

where $\mu=1$ as a consequence of (6) and the normalization $\sum_{j \in Q^{\prime}} \eta_{i j}=1$.

On the other hand, the probabilities in $P^{\prime}$ are tied by the set of normalizations (5). This means that, when minimizing with respect to parameter $p_{m}^{\prime}\left(\sigma, j_{1}, \ldots, j_{m}\right)$, a Lagrange multiplier $\nu_{\delta_{m}^{\prime}}\left(\sigma, j_{1}, \ldots, j_{m}\right)$ has to be included. In this case, one gets

$$
\begin{aligned}
& p_{m}^{\prime}\left(\sigma, j_{1}, j_{2}, \ldots, j_{m}\right)=\frac{1}{\nu_{\delta_{m}^{\prime}}\left(\sigma, j_{1}, j_{2}, \ldots, j_{m}\right)} \\
& \sum_{i_{1}, i_{2}, \ldots, i_{m} \in Q} C_{\delta_{m}\left(\sigma, i_{1}, i_{2}, \ldots, i_{m}\right)} p_{m}\left(\sigma, i_{1}, i_{2}, \ldots, i_{m}\right) \eta_{i_{1} j_{1}} \eta_{i_{2} j_{2}} \cdots \eta_{i_{m} j_{m}}
\end{aligned}
$$

The normalization (5), together with (20), entails that $\nu_{j}=\sum_{i \in Q} C_{i} \eta_{i j}$.

It is worth remarking that (23) and (24) are also valid in case $A$ is any given nondeterministic tree automaton provided that (20) and (21) are suitably rewritten.

As an illustration, Tables 1 and 2 describe a $k$-testable automaton $A^{\prime}$ with $k=3$ that approximates the (non- $k$-testable) PDTA with $\Delta$ defined in (16) and the following transition probabilities

$$
\begin{aligned}
\rho\left(q_{1}\right) & =1, \rho\left(q_{2}\right)=0, \\
p_{0}(a) & =p_{0}(b)=0.75, \\
p_{2}\left(a, q_{1}, q_{2}\right) & =p_{2}\left(a, q_{1}, q_{1}\right)=0.25 .
\end{aligned}
$$

Table 3 shows how the relative entropy between the original model and the approximate one is small compared to the entropy of the automaton (in this example, $H(A)=G(A, A)=1.6226$ bits). The KullbackLeibler divergence or relative entropy between the exact model $A$ and the approximate one $A^{\prime}$ is computed using (18) to (21). As expected, the quality of the approximation
TABLE 2

Probabilities in $P^{\prime}$ for the Approximate 3-Testable Model after the Same Example Automaton $A$

\begin{tabular}{l|l}
\hline$p_{0}(a)=1$ & $p_{0}(b)=1$ \\
\hline$p_{2}(a, a, b)=0.75$ & $p_{2}(a, a, a)=0.2812$ \\
$p_{2}(a, a(a b), b)=0.1875$ & $p_{2}(a, a(a b), a)=0.07031$ \\
$p_{2}(a, a(a a), b)=0.0625$ & $p_{2}(a, a(a a), a)=0.02344$ \\
\hline$p_{2}(a, a, a(a a))=0.3984$ & $p_{2}(a, a, a(a b))=0.07031$ \\
$p_{2}(a, a(a b), a(a a))=0.0996$ & $p_{2}(a, a(a b), a(a b))=0.0175$ \\
$p_{2}(a, a(a a), a(a a))=0.0332$ & $p_{2}(a, a(a a), a(a b))=0.0059$ \\
\hline
\end{tabular}

Each probability corresponds to a tree in Fig. 4. The probabilities in the same block add up to 1.

TABLE 3

Kullback-Leibler Divergence (Bits) in the Example between the Approximate Automaton $A^{\prime}$ and the Original One $A$ (Whose Entropy Is $H(A)=1.6226)$ for Different Values of $k$

\begin{tabular}{rrr}
\hline$k$ & $H\left(A, A^{\prime}\right)$ & $\left|\Delta^{\prime}\right|$ \\
\hline 2 & 0.348687 & 4 \\
3 & 0.165707 & 14 \\
4 & 0.021793 & 100 \\
5 & 0.010357 & 7102 \\
\hline
\end{tabular}

The second column shows the number of transitions in $\Delta^{\prime}$, that is, the size of the set $\Theta^{[k]}$.

improves as $k$ increases at the expense of a considerable growth in the number of states so that moderate values of $k$ are to be preferred.

\section{Learning Stochastic Strictly Locally Testable Tree Languages}

A number of algorithms have been proposed in the past to build automata from stochastic samples. In some cases, the result is a nondeterministic automaton [17], [32]; in others, the automata are deterministic [33], [34]. Here, a stochastic sample $\Omega=\tau_{1}, \tau_{2}, \ldots \tau_{|\Omega|}$ consists of a sequence of (possibly repeated) trees generated according to an unknown probability distribution and a procedure to learn a probabilistic model from $\Omega$ can be derived using the results in former section.

Given a stochastic sample $\Omega$, we will denote with $\hat{\Omega}$ the set of (different) trees in $\Omega$. It is always possible to build a trivial PDTA $A$ such that, for every tree $t$ in the sample, $p(t \mid A)$ coincides with the relative frequency of $t$ in the sample as follows:

$$
\begin{aligned}
& Q=\operatorname{sub}(\hat{\Omega}), \\
& \delta_{m}\left(\sigma, t_{1}, \ldots, t_{m}\right)= \begin{cases}\sigma\left(t_{1} \cdots t_{m}\right) & \text { if } \sigma\left(t_{1} \cdots t_{m}\right) \in Q, \\
\perp & \text { otherwise, }\end{cases} \\
& p_{m}\left(\sigma, t_{1}, \ldots, t_{m}\right)=1 \text { for all } \sigma\left(t_{1} \cdots t_{m}\right) \in Q, \\
& \rho(t)=\frac{1}{|\Omega|} \sum_{n=1}^{|\Omega|} D\left(t, \tau_{n}\right) .
\end{aligned}
$$

Note that $\delta(t)=t$ for all trees in the sample. 
On the other hand, as described after (13), the minimal $k$-testable model $A^{\prime}$ recognizing $\hat{\Omega}$ has states $Q^{\prime}=\Theta^{[k-1]}$ and transitions $\delta_{m}^{\prime}\left(\sigma, t_{1}, \ldots, t_{m}\right)=r_{k-1}\left(\sigma\left(t_{1} \cdots t_{m}\right)\right)$ with

$$
\Theta^{[k]}=\bigcup_{n=1}^{|\Omega|} f_{k}\left(\tau_{n}\right) \cup s_{k-1}\left(\tau_{n}\right) .
$$

Then, $\delta^{\prime}(t)=r_{k-1}(t)$ for all $t \in \hat{\Omega}$.

In order to find the probabilities $\rho^{\prime}$ in the corresponding PDTA, one should note that, in this case, $\eta_{i j}$ is 1 if $r_{k-1}(i)=j$ and zero otherwise. Then, using (23), one gets

$$
\begin{aligned}
\rho^{\prime}(j) & =\frac{1}{|\Omega|} \sum_{n=1}^{|\Omega|} \sum_{i \in Q} D\left(r_{k-1}(i), j\right) D\left(i, \tau_{n}\right) \\
& =\frac{1}{|\Omega|} \sum_{n=1}^{|\Omega|} D\left(j, r_{k-1}\left(\tau_{n}\right)\right) .
\end{aligned}
$$

Finally, in order to obtain $P^{\prime}$, one should take into account that, in this case, $C_{i}=\frac{1}{|\Omega|} \sum_{n} \Phi\left(i, \tau_{n}\right)$, where $\Phi(t, \tau)$ counts the number of nodes in $\tau$ that expand a subtree $s$ such that $s=\tau$. Then, one gets, for the denominator in (24),

$$
\frac{1}{|\Omega|} \sum_{n=1}^{|\Omega|} E^{[k-1]}\left(\sigma\left(t_{1} \cdots t_{m}\right), \tau_{n}\right)
$$

where

$$
E^{[k-1]}\left(j, \tau_{n}\right)=\sum_{i \in Q} \Phi\left(i, \tau_{n}\right) D\left(r_{k-1}(i), r_{k-1}(j)\right)
$$

counts the number of nodes in $\tau_{n}$ that expand a subtree $s$ such that $\delta^{\prime}(s)=\delta^{\prime}(j)$.

On the other hand, $r_{k-1}\left(i_{1}\right)=j_{1}, \ldots$ and $r_{k-1}\left(i_{m}\right)=j_{m}$ if and only if $r_{k}\left(\sigma\left(i_{1}, \ldots, i_{m}\right)\right)=\sigma\left(j_{1}, \ldots, j_{m}\right)$. Taking this into account, one gets, for the numerator in (24),

$$
\begin{aligned}
& \frac{1}{|\Omega|} \sum_{n=1}^{|\Omega|} \sum_{i_{1} \cdots i_{m} \in Q} \\
& \Phi\left(\sigma\left(i_{1} \cdots i_{m}\right), \tau_{n}\right) D\left(r_{k}\left(\sigma\left(i_{1} \cdots i_{m}\right)\right), \sigma\left(j_{1} \cdots j_{m}\right)\right)
\end{aligned}
$$

and, therefore,

$$
p_{m}^{\prime}\left(\sigma, j_{1}, \ldots, j_{m}\right)=\frac{\sum_{n} E^{[k]}\left(\sigma\left(j_{1} \cdots j_{m}\right), \tau_{n}\right)}{\sum_{n} E^{[k-1]}\left(\sigma\left(j_{1} \cdots j_{m}\right), \tau_{n}\right)} .
$$

As expected, (27) and (31) show that the closest automaton $A^{\prime}$ to the sample $\Omega$ is obtained when each transition is assigned a probability that is proportional to the number of times the transition is performed when processing the sample. In practice, it is useful to store the probabilities given by these equations as pairs (numerator, denominator), so that, if a new tree $\tau$ is added to the sample $\Omega$, the automaton $A^{\prime}$ can be easily updated to account for the additional information. For this incremental update, it suffices to increment each term in the pair with the partial sums obtained for $\tau$.

In our example with $k=3$, presented at the end of Section 3, where $\tau=a(a(a(a b)) b$ ), the relevant values (see Fig. 3) are:

$$
\begin{gathered}
E^{[3]}(a(a(a) b), \tau)=E^{[3]}(a(a(a b)), \tau) \\
=E^{[3]}(a(a b), \tau)=E^{[3]}(a, \tau)=1 \\
E^{[3]}(b, \tau)=2 \\
E^{[2]}(a(a), \tau)=E^{[2]}(a, \tau)=1 \\
E^{[2]}(a(a(a) b), \tau)=E^{[2]}(a(a b), \tau)=E^{[2]}(b, \tau)=2 .
\end{gathered}
$$

Then, one gets for the five transitions involved:

$$
\begin{aligned}
& \left.p_{2}(a, a(a), b)\right)=p_{2}(a, a, b)=1 / 2, \\
& p_{0}(a)=p_{0}(b)=p_{1}(a, a(a b))=1,
\end{aligned}
$$

and $\rho\left(q_{2}\right)=1$. All other probabilities are zero. If we compute, for instance, the probability of $a(a(a(a b))) b)$ with this model, the result is $1 / 4$.

\section{Smoothed Offspring Annotated Models of SYNTACTICAL STRUCTURE}

Context-free grammars may be considered the customary way of representing syntactical structure in natural language sentences. A set of rather radical hypotheses as to how humans select the best parse tree [35] proposes that a great deal of syntactic disambiguation may actually occur without the use of any semantic information, that is, just by selecting a preferred parse tree. It may be argued that the preference of a parse tree with respect to another is largely due to the relative frequencies with which those choices have led to a successful interpretation. This sets the ground for a family of techniques which use a probabilistic scoring of parses to find the correct parse in each case.

Probabilistic scorings depend on parameters which are usually estimated from data, that is, from parsed text corpora such as the Penn Treebank [36]. The most straightforward approach is that of treebank grammars [37]. Treebank grammars are probabilistic context-free grammars (PCFG) in which the probability that a particular nonterminal is expanded according to a given rule is estimated as the relative frequency of that expansion by simply counting the number of times it appears in a manually parsed corpus. Our probabilistic $k$-testable models can be regarded as offspring-annotated models where the expansion probabilities are dependent on the future expansions of children and include treebank grammars as a special case. Other models, such as Johnson's [38] parent-annotated models (or, more generally, ancestor annotated models) and IBM history-based grammars [23, p. 423], [39] or Bod and Scha's Data-Oriented parsing [40] offer an alternate approach in which the probability of expansion of a given nonterminal is made dependent on the previous expansions. An interesting property of many of these models is that, even though they may be seen as context-dependent, they may still be easily rewritten as context-free models in terms of specialized versions of the original nonterminals.

For instance, if $\Omega=\tau_{1}, \tau_{2}, \ldots, \tau_{|\Omega|}$ is a treebank, that is, a stochastic sample of parse trees, the alphabet $\Sigma$ can be safely partitioned into the subset $s_{1}(\hat{\Omega})$ of labels that may only appear at leaves (usually lexical forms or part of speech tags) and its complementary subset $\Sigma-s_{1}(\hat{\Omega})$ of labels at internal nodes (syntactic tags). Then, we can define a probabilistic $k$-testable grammar as $G^{[k]}=\left(V^{[k]}, T, I, R^{[k]}, p^{[k]}\right)$, where $V^{[k]}=$ $\{I\} \cup r_{k-1}\left(f_{k}(\hat{\Omega}) \cup s_{k-1}(\hat{\Omega})\right)-s_{1}(\hat{\Omega})$ is the set of nonterminals, $T=s_{1}(\hat{\Omega})$ is the set of terminals, $I$ is the start symbol, $R^{[k]}$ is the 


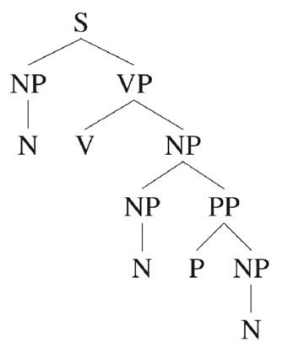

Fig. 5. An example of parse tree.

set of production rules, and $p^{[k]}$ a probability function, builts as follows:

- $\quad$ For every tree $t \in r_{k-1}(\hat{\Omega})$, add the rule $I \rightarrow t$ to $R^{[k]}$ and compute its probability as

$$
p^{[k]}(I \rightarrow t)=\frac{1}{|\Omega|} \sum_{n=1}^{|\Omega|} D\left(t, r_{k-1}\left(\tau_{n}\right)\right) .
$$

- $\quad$ For every tree $\sigma\left(t_{1} t_{2} \cdots t_{m}\right) \in f_{k}(\hat{\Omega})$, add the rule $r_{k-1}$ $\left(\sigma\left(t_{1} t_{2} \cdots t_{m}\right)\right) \rightarrow t_{1} t_{2} \ldots t_{m}$ to $R^{[k]}$ and compute its probability as

$$
\begin{aligned}
& p^{[k]}\left(r_{k-1}\left(\sigma\left(t_{1} t_{2} \cdots t_{m}\right)\right) \longrightarrow t_{1} t_{2} \ldots t_{m}\right) \\
& =\frac{\sum_{n} E^{[k]}\left(\sigma\left(t_{1} t_{2} \cdots t_{m}\right), \tau_{n}\right)}{\left.\sum_{n} E^{[k-1]}\left(\sigma\left(t_{1} t_{2} \cdots t_{m}\right)\right), \tau_{n}\right)} .
\end{aligned}
$$

- For every tree $\sigma\left(t_{1} t_{2} \ldots t_{m}\right) \in s_{k-1}(\hat{\Omega})-T$, add the rule $\sigma\left(t_{1} t_{2} \cdots t_{m}\right) \rightarrow t_{1} t_{2} \ldots t_{m}$ to $R^{[k]}$, its probability being one:

$$
p^{[k]}\left(\sigma\left(t_{1} t_{2} \ldots t_{m}\right) \longrightarrow t_{1} t_{2} \ldots t_{m}\right)=1 .
$$

The above rules and probabilities are analogous to those given by (27) and (31) and define a consistent probabilistic context-free grammar. ${ }^{5}$ With this definition, when $k=2$, only the label of the node is taken into account and the $k$-testable model coincides with the simple rule-counting approach used in treebank grammars [37]. As an illustration, consider a tiny sample containing only the parse tree $t$ depicted in Fig. 5. Then, $r_{1}(t)=\mathrm{S}, f_{2}(t)=\{\mathrm{S}(\mathrm{NP} \mathrm{VP}), \mathrm{NP}(\mathrm{N}), \mathrm{VP}(\mathrm{VNP}), \mathrm{NP}$ $(\mathrm{NPPP}), \mathrm{PP}(\mathrm{P} \mathrm{NP})\}, s_{1}(t)=\{N, V, P\}$, and the PCFG is $G^{[2]}$ $=\left(\{\mathrm{I}, \mathrm{S}, \mathrm{NP}, \mathrm{VP}, \mathrm{PP}\},\{\mathrm{N}, \mathrm{V}, \mathrm{P}\}, \mathrm{I}, R^{[2]}, p^{[2]}\right)$ with rules and probabilities

$$
\begin{aligned}
& \mathrm{I} \rightarrow \mathrm{S} \quad \text { (1) } \\
& \mathrm{S} \rightarrow \mathrm{NP} \text { VP }(1) \\
& \mathrm{NP} \rightarrow \mathrm{NP} P P \quad(0.25) \\
& \mathrm{NP} \rightarrow \mathrm{N} \\
& \mathrm{VP} \rightarrow \mathrm{V} \mathrm{NP} \\
& \mathrm{PP} \rightarrow \mathrm{P} \mathrm{NP}
\end{aligned}
$$

When $k=3$, one gets a child-annotated model, that is, nonterminal symbols are of the form $\sigma\left(\sigma_{1} \sigma_{2} \ldots \sigma_{m}\right)$. In our example, $r_{2}(t)=\mathrm{S}(\mathrm{NP}$ VP $), f_{3}(t)=\{\mathrm{S}(\mathrm{NP}(\mathrm{N}) \mathrm{VP}(\mathrm{V} \mathrm{NP})), \mathrm{VP}$ $(\mathrm{V} N \mathrm{~N}(\mathrm{NP} P \mathrm{P})), \mathrm{NP}(\mathrm{NP}(\mathrm{N}) \mathrm{PP}(\mathrm{P} \mathrm{NP})), \mathrm{PP}(\mathrm{P} \mathrm{NP}(\mathrm{N}))\}, s_{2}(t)$ $=\{\mathrm{NP}(\mathrm{N}), \mathrm{N}, \mathrm{V}, \mathrm{P}\}$, and the PCFG is $G^{[3]}=(\{\mathrm{I}, \mathrm{S}(\mathrm{NP} V \mathrm{P})$,
$\mathrm{NP}(\mathrm{N}), \mathrm{VP}(\mathrm{V} N \mathrm{P}), \mathrm{NP}(\mathrm{NP} P \mathrm{P}), \mathrm{PP}(\mathrm{P} \mathrm{NP})\}, \quad\{\mathrm{N}, \mathrm{V}, \mathrm{P}\}$, $\left.\mathrm{I}, R^{[3]}, p^{[3]}\right)$ with rules and probabilities

$$
\begin{aligned}
\mathrm{I} & \longrightarrow \mathrm{S}(\mathrm{NP} \mathrm{VP}) \\
\mathrm{S}(\mathrm{NP} \mathrm{VP}) & \longrightarrow \mathrm{NP}(\mathrm{N}) \mathrm{VP}(\mathrm{V} \mathrm{NP}) \\
\mathrm{VP}(\mathrm{VNP}) & \longrightarrow \mathrm{VP}(\mathrm{NP} P \mathrm{PP}) \\
\mathrm{NP}(\mathrm{NP} P \mathrm{P}) & \longrightarrow \mathrm{NP}(\mathrm{N}) \mathrm{PP}(\mathrm{P} \mathrm{NP}) \\
\mathrm{PP}(\mathrm{PNP}) & \longrightarrow \mathrm{P} \mathrm{NP}(\mathrm{N}) \\
\mathrm{NP}(\mathrm{N}) & \longrightarrow \mathrm{N}
\end{aligned}
$$

In general, $k$-testable grammars with larger values of $k$ contain more specialized rules and, therefore, are less ambiguous and allow for faster parsing. In contrast, typical treebank grammars have 100 percent coverage (as remarked in [37]) unlike with higher order grammars where sentences without a valid parse tree are not uncommon. Therefore, the use of smoothing techniques becomes necessary if one wants to use these models for parsing. Two classical techniques of this type are linear interpolation and backing-off [9].

Smoothing through linear interpolation is performed by computing the probability of events as a weighted average of the probabilities given by different models. For instance, the smoothed probability of a $k$-testable model can be computed as a weighted average of the probability given by the grammar $G^{[k]}$ and that given by the grammar $G^{[k-1]}$ :

$$
p\left(t \mid G_{\mathrm{INT}}^{[k]}\right)=(1-\lambda) p\left(t \mid G^{[k]}\right)+\lambda p\left(t \mid G^{[k-1]}\right) .
$$

In turn, $p\left(t \mid G^{[k-1]}\right)$ can be replaced by its smoothed version. The parameter $\lambda \in[0,1]$ can be chosen to maximize the likelihood of the sample. In practice, linear interpolation can be slow as it is dominated by the lower-order, more ambiguous, parse.

In contrast, backing-off allows one to avoid lower-order parsing when possible:

$$
p^{[k]}\left(t \mid G_{\text {TREE }}^{[k]}\right)= \begin{cases}(1-\lambda) p\left(t \mid G^{[k]}\right) & \text { if } p\left(t \mid G^{[k]}\right)>0 \\ \frac{\lambda}{\Lambda} p\left(t \mid G^{[k-1]}\right) & \text { otherwise, }\end{cases}
$$

where $\Lambda$ is a normalization factor that can be computed as

$$
\Lambda=1-\sum_{t: p\left(t \mid G^{[k]}\right)>0} p\left(t \mid G^{[k-1]}\right) .
$$

If the parameter $\lambda$ is small, this approach essentially parses the sentence with the higher-order grammar unless no parse tree is provided by this grammar. Because only in such a case is the lower-order model called, backing-off is faster than linear interpolation. However, the lack of a single rule in the sample can force the parser to use the lower-order model and then loose all the higher-order information for a whole sentence.

Here, we introduce an alternative approach: the use of rule-based backing-off. In this approach, the set of rules is generalized with the probabilities

$$
\begin{aligned}
& p\left(\left(r_{k-1}\left(\sigma\left(t_{1} \cdots t_{m}\right)\right) \rightarrow t_{1} t_{2} \cdots t_{m} \mid G_{\mathrm{RULE}}^{[k]}\right)=\right. \\
& \left\{\begin{array}{c}
(1-\lambda) p^{[k]}\left(r_{k-1}\left(\sigma\left(t_{1} \cdots t_{m}\right)\right) \rightarrow t_{1} t_{2} \cdots t_{m}\right) \\
\quad \text { if } p^{[k]}\left(r_{k-1}\left(\sigma\left(t_{1} \cdots t_{m}\right)\right) \rightarrow t_{1} t_{2} \cdots t_{m}\right)>0 \\
\frac{\lambda}{\Lambda\left(r_{k-1}\left(\sigma\left(t_{1} \cdots t_{m}\right)\right)\right)} \prod_{i: r_{k-2}\left(t_{i}\right) \neq t_{i}} p^{[k-1]}\left(r_{k-2}\left(t_{i}\right) \rightarrow t_{i}\right) \text { otherwise, }
\end{array}\right.
\end{aligned}
$$




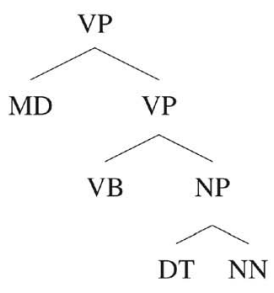

(a)

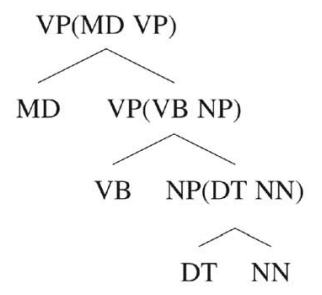

(b)

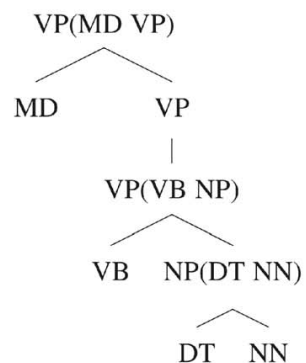

(c)

Fig. 6. (a) Parse tree obtained with $G^{[2]}$, (b) parse tree obtained with $G^{[3]}$, invalid if the rule $\operatorname{VP}(\mathrm{MDVP}) \rightarrow \operatorname{MD} \mathrm{VP}(\mathrm{VBNP})$ is missing in $R^{[3]}$, and (c) parse tree obtained with $G^{\prime}$, where backoff rules $\mathrm{VP}(\mathrm{MDVP}) \rightarrow \mathrm{MD}$ VP and VP $\rightarrow \mathrm{VP}(\mathrm{VBNP})$ have been applied.

where $t_{1}, \ldots, t_{m}$ are parse trees and

$$
\begin{aligned}
& \Lambda\left(r_{k-1}\left(\sigma\left(t_{1} \cdots t_{m}\right)\right)\right)= \\
& 1-\sum_{r_{k-1}\left(\sigma\left(t_{1} \cdots t_{m}\right)\right) \rightarrow \tau_{1} \cdots \tau_{m} \in R^{[k]}} \prod_{i: r_{k-2}\left(\tau_{i}\right) \neq \tau_{i}} p^{[k-1]}\left(r_{k-2}\left(\tau_{i}\right) \rightarrow \tau_{i}\right) .
\end{aligned}
$$

Using this rule-based backing-off requires the implementation of specific parsers as building the whole grammar is unfeasible due to the large number of implicit rules (even if only those assigned a strictly positive probability in the last case of (35) are considered). An alternative scheme that requires only minor modifications is using a quasi-equivalent grammar $G^{\prime}$ built as follows:

1. Add the rules in $R^{[k]}$ to $R^{\prime}$ with probabilities

$$
p^{\prime}(X \rightarrow \alpha)=(1-\lambda) p^{[k]}(X \rightarrow \alpha) .
$$

2. For every variable $\sigma\left(t_{1} t_{2} \cdots t_{m}\right)$ in $V^{[k]}$, add a rule $\sigma\left(t_{1} t_{2} \cdots t_{m}\right) \rightarrow t_{1} t_{2} \ldots t_{m}$ to $R^{\prime}$ whose probability is

$$
p^{\prime}\left(\sigma\left(t_{1} t_{2} \cdots t_{m}\right) \rightarrow t_{1} t_{2} \ldots t_{m}\right)=\frac{\lambda}{\Lambda\left(\sigma\left(t_{1} \cdots t_{m}\right)\right)} .
$$

3. For every rule $r_{k-2}\left(\sigma\left(t_{1} t_{2} \cdots t_{m}\right)\right) \rightarrow t_{1} t_{2} \cdots t_{m}$ in $R^{[k-1]}$, add $r_{k-2}\left(\sigma\left(t_{1} t_{2} \cdots t_{m}\right)\right) \rightarrow r_{k-1}\left(\sigma\left(t_{1} t_{2} \cdots t_{m}\right)\right)$ to $R^{\prime}$ and the probability

$$
\begin{aligned}
& p^{\prime}\left(r_{k-2}\left(\sigma\left(t_{1} t_{2} \cdots t_{m}\right)\right) \rightarrow r_{k-1}\left(\sigma\left(t_{1} t_{2} \cdots t_{m}\right)\right)\right)= \\
& p^{[k-1]}\left(r_{k-2}\left(\sigma\left(t_{1} t_{2} \cdots t_{m}\right)\right) \rightarrow t_{1} t_{2} \cdots t_{m}\right) .
\end{aligned}
$$

As shown in Fig. 6, the parse trees generated by this grammar $G^{\prime}$ and their probabilities are, after an adequate projection $\zeta$, identical to those generated by using (35): If a node with label $X$ has as single descendent a subtree $Y(\alpha)$ and the depth of label $X$ is smaller than that of label $Y$, the projection operation $\zeta$ has to be applied so that $\zeta(X(Y(\alpha)))=Y(\alpha)$. Then, $G^{\prime}$ can be used with a standard chart parser provided that some care is taken to avoid selecting a projected subtree whenever the same subtree can be obtained without projection. This can be checked by redefining the suitable comparison operator so that $t_{1}$ is not a better tree than $t_{2}$ if $\zeta\left(t_{1}\right)=\zeta\left(t_{2}\right)=t_{2}$ (even if $p\left(t_{1} \mid G^{\prime}\right)>p\left(t_{2} \mid G^{\prime}\right)$.

\section{EXPERIMENTS}

\subsection{General Conditions}

We have performed a series of experiments to assess the structural disambiguation performance of offspring-annotated models as compared to standard treebank grammars, that is, to compare their relative ability for selecting the best parse tree. To better put these comparisons in context, we have also evaluated Johnson's [38] parent annotation scheme. To build training corpora and test sets of parse trees, we have used English parse trees from the Penn Treebank, release 3, with small, basically structure-preserving modifications (consistently with previous work [37], [38]):

- insertion of a root node, $I=\mathrm{ROOT}$, in all sentences, to encompass the sentence and final periods, etc.,

- removal of nonsyntactic annotations (prefixes and suffixes) from constituent labels (for instance, NP-SBJ is reduced to NP),

- removal of empty constituents, and

- collapse of single-child nodes with the parent node when they have the same label. ${ }^{6}$

In all experiments, the training corpus consisted of all of the trees $(41,532)$ in sections 02 to 22 of the Wall Street Journal portion of Penn Treebank, modified as above. This gives a total number of more than 600,000 subtrees. The test set contained all sentences in Section 23 having less than 40 words.

All grammar models were written as standard context-free grammars and a chart parser [43] was used to obtain the most likely parse for each sentence in the training set. This parse was compared to the corresponding gold-standard tree in the test set using the customary PARSEVAL evaluation metric [22], [23, p. 432] after deannotating the most likely tree delivered by the parser. PARSEVAL gives partial credit to incorrect parses by establishing three measures:

- Labeled precision $(P)$ is the fraction of correctly labeled nonterminal bracketing (constituents) in the most likely parse which match the gold-standard parse,

- Labeled recall $(R)$ is the fraction of brackets in the gold-standard parse which are found in the most likely parse with the same label, and

- Crossing brackets $(X)$ refers to the fraction of constituents in one parse cross over constituent boundaries in the other parse.

6. As most cases are labeling mistakes. 

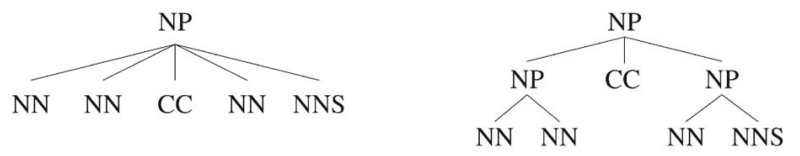

Fig. 7. Flat and structured parse of a sentence.

The crossing brackets measure does not take constituent labels into account and will not be shown here. Some authors (see, e.g., [44]) have questioned partial-credit evaluation metrics such as the PARSEVAL measures. In particular, if one wants to use a probability model to perform structural disambiguation before assigning some kind of interpretation to the parsed sentence, it may well be argued that the exact match between the gold-standard tree and the most likely tree is the only possible relevant measure. It is, however, known that the Penn Treebank, even in its release 3, still suffers from problems. One of the problems worth mentioning (discussed in detail by Krotov et al. [45]) is the presence of far too many partially bracketed constructs according to rules like NP $\rightarrow$ NN NN CC NN NNS, which lead to very flat trees, when one can, in the same treebank, find rules such as $\mathrm{NP} \rightarrow \mathrm{NN} \mathrm{NN}, \mathrm{NP} \rightarrow \mathrm{NN} \mathrm{NNS}$, and $\mathrm{NP} \rightarrow \mathrm{NP} \mathrm{CC} \mathrm{NP}$, which would lead to more structured parses, such as that depicted in Fig. 7.

Some of these flat parses may indeed be too flat to be useful for semantic purposes and have little linguistic plausibility; therefore, if one gets a more refined parse, one may consider it to be the one leading to the correct interpretation or not, but it surely contains more information than the flat, unstructured one. For this reason, we have chosen to give, in addition to the exact-match figure, the percentage of trees having 100 percent recall because these are the trees in which the most likely parse is either exactly the gold-standard parse or a refinement thereof in the sense of the previous example.

\subsection{Structural Disambiguation Results}

The models which were evaluated are the following:

1. a standard treebank grammar[37], with no annotation of node labels (15,140 rules),

2. a parent-annotated grammar [38], with information at each node of the parent's label (23,020 rules),

3. a linear interpolation of $k=3$ and $k=2 k$-testable grammars with parameter $\lambda=0.7$ selected to maximize the likelihood of the sample,

4. a 3-testable grammar (92,830 rules) smoothed with tree-based backing-off, and

5. a 3-testable grammar smoothed with rule-based backing-off (adding 10,250 rules of the second type described at the end of former section) with parameter $\lambda=0.005$ selected to maximize the sum of recall and precision.

As expected, the number of rules in the model increases as more information is conveyed by the node label, although this increase is not extreme. On the other hand, as the generalization power decreases, some sentences in the test would become unparsable by the $k$-testable grammar if the unsmoothed model was used. For instance, in our experiments, the child-annotated model was able to parse 94.6 percent of the sentences.

The results in Table 4 show that the best parsing performance is obtained using a child-annotated grammar
TABLE 4

Parsing Results with Different Grammars: Labeled Recall $R$, Labeled Precision $P$, Fraction of Sentences with Total Labeled Recall $f_{R=100 / \%}$, Fraction of Exact Matches

\begin{tabular}{lrrrr}
\hline Grammar & $R$ & $P$ & $f_{R=100 \%}$ & EXACT \\
\hline 1 (Treebank grammar) & $70.7 \%$ & $76.1 \%$ & $10.4 \%$ & $10.0 \%$ \\
2 (Parent annotated) & $80.0 \%$ & $81.9 \%$ & $18.5 \%$ & $16.3 \%$ \\
3 (Linear interpolation) & $80.2 \%$ & $78.6 \%$ & $21.7 \%$ & $17.4 \%$ \\
4 (Tree-based back-off) & $78.9 \%$ & $74.2 \%$ & $19.4 \%$ & $17.1 \%$ \\
5 (Rule-based back-off) & $82.4 \%$ & $81.3 \%$ & $25.7 \%$ & $17.5 \%$ \\
\hline
\end{tabular}

with a rule-based backing-off. The results obtained with parent-annotated models are comparable (except for 100 percent recall). However, parsers using child-annotated grammars are much faster because the number of possible parse trees considered is drastically reduced.

It may be worth mentioning that parse trees produced by child-annotated models tend to be more structured and refined than parent-annotated and unannotated parses, which tend to use rules that lead to flat trees in the sense mentioned above. Favoring structured parses may be convenient in some applications (especially where meaning has to be compositionally computed), but may be less convenient than flat parses when the structure obtained is incorrect.

\section{Conclusions}

We have introduced a class of probabilistic models (the strictly $k$-testable class) that describes tree languages and can be easily estimated from treebank samples. We have found an efficient procedure to compute the probabilities of the $k$-testable model that approximates any given stochastic rational tree language. The relative entropy between the $k$-testable model and the original one can also be efficiently computed and, as expected, the divergence becomes smaller as $k$ increases. These strictly locally testable models can be updated incrementally and are adequate to apply backing-off techniques [9] or to be used as starting point by more complex learning algorithms [34], [46].

Fixed-depth probabilistic models may be seen as a special PCFG in which nonterminals are specialized for each possible offspring (up to a certain depth) and, so, are the expansion probabilities of rules. This may help model linguistic phenomena such as the differential distribution of determinants in noun phrases acting like a subject or like an object. We have introduced a procedure to build $k$-testable grammars smoothed by using a rule-based backing-off approach. In particular, we have compared the parsing performance of these models with that of unannotated PCFG and of parentannotated PCFG [38]. As future work, we plan to study the use of statistical confidence criteria to eliminate unnecessary annotations by merging states, therefore, reducing the number of parameters to be estimated.

\section{ACKNOWLEDGMENTS}

The authors would like to thank Mikel L. Forcada for his useful suggestions and help with linguistic insight. This 
work was supported by the Spanish Comisión Interministerial de Ciencia y Tecnología through grants TIC200308496-C04 and TIC2003-08681-C02-01.

\section{REFERENCES}

[1] G. Riccardi, R. Pieraccini, and E. Bocchieri, "Stochastic Automata for Language Modeling," Computer Speech and Language, vol. 10, no. 4, pp. 265-293, 1996.

[2] J. Hu, W. Turin, and M.K. Brown, "Language Modeling with Stochastic Automata," Proc. Fourth Int'l Conf. Spoken Language Processing, vol. 1, pp. 406-409, 1996, http://www.asel.udel.edu/ icslp/cdrom/vol1/996/a996.pdf.

[3] Y. Esteve, F. Bechet, A. Nasr, and R.D. Mori, "Stochastic Finite State Automata Language Model Triggered by Dialogue States," Proc. Eurospeech, pp. 725-728, 2001.

[4] R. McNaughton and S. Papert, Counter-Free Automata. Cambridge, Mass.: MIT Press, 1971.

[5] P. García and E. Vidal, "Inference of $k$-Testable Languages in the Strict Sense and Application to Syntactic Pattern Recognition," IEEE Trans. Pattern Analysis and Machine Intelligence, vol. 12, no. 9, pp. 920-925, Sept. 1990.

[6] T. Yokomori, "On Polynomial-Time Learnability in the Limit of Strictly Deterministic Automata," Machine Learning, vol. 19, no. 2, pp. 153-179, 1995.

[7] T. Yokomori, N. Ishida, and S. Kobayashi, "Learning Local Languages and Its Application to Protein Alpha-Chain Identification," Proc. 27th Hawaii Int'l Conf. System Sciences, pp. 113-122, 1994, citeseer.ist.psu.edu/article/yokomori96learning.html.

[8] K.W. Church and W. Gale, "A Comparison of the Enhanced Good-Turing and Deleted Estimation Methods for Estimating Probabilities of English Bigrams," Computer Speech and Language, vol. 5, pp. 19-54, 1991.

[9] H. Ney, U. Essen, and R. Kneser, "On the Estimation of Small Probabilities by Leaving-One-Out," IEEE Trans. Pattern Analysis and Machine Intelligence, vol. 17, no. 12, pp. 1202-1212, Dec. 1995.

[10] E. Charniak, Statistical Language Learning. MIT Press, 1993.

[11] K. Sima'an, R. Bod, S. Krauwer, and R. Scha, "Efficient Disambiguation by Means of Stochastic Tree Substitution Grammars," Proc. Int'l Conf. New Methods in Language Processing, D.B. Jones and H.L. Somers, eds., pp. 50-58, Sept. 1994.

[12] A. Stolcke, "An Efficient Context-Free Parsing Algorithm that Computes Prefix Probabilities," Computational Linguistics vol. 21, no. 2, pp. 165-201, 1995.

[13] M. Thorup, "Disambiguating Grammars by Exclusion of SubParse Trees," Acta Informatica, vol. 33, no. 6, pp. 511-522, 1996.

[14] P. Prescod, "Formalizing XML and SGML Instances with Forest Automata Theory," technical report, draft technical paper, Dept. of Computer Science, Univ. of Waterloo, Waterloo, Ontario, Canada, 2002.

[15] M. Murata, "Transformation of Documents and Schemas by Patterns and Contextual Conditions," Proc. Principles of Document Processing, Third Int'l Workshop, C.K. Nicholas and D. Wood, eds., vol. 1293, pp. 153-169, 1997.

[16] J. Hopcroft and J.D. Ullman, Introduction to Automata Theory, Language, and Computation. Reading, Mass.: Addison-Wesley, 1979.

[17] Y. Sakakibara, M. Brown, R.C. Underwood, I.S. Mian, and D. Haussler, "Stochastic Context-Free Grammars for Modeling RNA," Proc. 27th Ann. Hawaii Int'l Conf. System Sciences, Vol. 5: Biotechnology Computing, L. Hunter, ed., pp. 284-294, Jan. 1994.

[18] F. Gécseg and M. Steinby, Tree Automata. Budapest: Akademiai Kiado, 1984.

[19] Y. Sakakibara, "Efficient Learning of Context-Free Grammars from Positive Structural Examples," Information and Computation, vol. 97, no. 1, pp. 23-60, Mar. 1992.

[20] M. Nivat and A. Podelski, "Minimal Ascending and Descending Tree Automata," SIAM J. Computing, vol. 26, no. 1, pp. 39-58, 1997.

[21] T. Knuutila, "Inference of $k$-Testable Tree Languages," Proc. Int'l Workshop Structural and Syntactic Pattern Recognition, Advances in Structural and Syntactic Pattern Recognition, H. Bunke, ed., 1993.

[22] E. Black, S. Abney, D. Flickinger, C. Gdaniec, R. Grishman, P. Harrison, D. Hindle, R. Ingria, F. Jelinek, J. Klavans, M. Liberman, M. Marcus, S. Roukos, B. Santorini, and T. Strzalkowski, "A Procedure for Quantitatively Comparing the Syntatic Coverage of English Grammars," Proc. Speech and Natural Language Workshop 1991, pp. 306-311, 1991.
[23] C. Manning and H. Schütze, Foundations of Statistical Natural Language Processing. Cambridge, Mass.: MIT Press, 1999.

[24] A. Radford, M. Atkinson, D. Britain, H. Clahsen, and A. Spencer, Linguistics: An Introduction. Cambridge, U.K.: Cambridge Univ. Press, 1999.

[25] A.V. Aho, R. Sethi, and J.D. Ullman, Compilers Principles, Techniques, and Tools. Addison Wesley, 1986.

[26] W.J. Hutchins and H.L. Somers, An Introduction to Machine Translation. New York: Academic Press, 1992.

[27] T.M. Cover and J.A. Thomas, Elements of Information Theory. New York: John Wiley \& Sons, 1991.

[28] C.S. Wetherell, "Probabilistic Languages: A Review and Some Open Questions," ACM Computing Surveys, vol. 12, no. 4, pp. 361379, Dec. 1980.

[29] E. Gold, "Language Identification in the Limit," Information and Control, vol. 10, pp. 447-474, 1967.

[30] A. Stolcke and J. Segal, "Precise $n$-Gram Probabilities from Stochastic Context-Free Grammars," Technical Report TR-94-007, Int'l Computer Science Inst., Berkeley, Calif., Jan. 1994.

[31] J. Calera-Rubio and R.C. Carrasco, "Computing the Relative Entropy between Regular Tree Languages," Information Processing Letters, vol. 68, no. 6, pp. 283-289, 1998.

[32] L.R. Rabiner, "A Tutorial on Hidden Markov Models and Selected Applications in Speech Recognition," Proc. IEEE, vol. 77, no. 2, pp. 257-285, Feb. 1989.

[33] R.C. Carrasco and J. Oncina, "LearningDeterministic Regular Grammars from Stochastic Samples in Polynomial Time," RAIRO (Theoretical Informatics and Applications), vol. 33, no. 1, pp. 1-20, 1999.

[34] R.C. Carrasco, J. Oncina, and J. Calera-Rubio, "Stochastic Inference of Regular Tree Languages," Machine Learning, vol. 44, nos. 1/2, pp. 185-197, 2001.

[35] L. Frazier and K. Rayner, "Making and Correcting Errors During Sentence Comprehension: Eye Movements in the Analysis of Structurally Ambiguous Sentences," Cognitive Psychology, vol. 14, pp. 178-210, 1982.

[36] M.P. Marcus, B. Santorini, and M. Marcinkiewicz, "Building a Large Annotated Corpus of English: The Penn Treebank," Computational Linguistics, vol. 19, pp. 313-330, 1993.

[37] E. Charniak, "Tree-Bank Grammars," Proc. 13th Nat'l Conf. Artificial Intelligence and the Eighth Innovative Applications of Artificial Intelligence Conf., pp. 1031-1036, 1996.

[38] M. Johnson, "PCFG Models of Linguistic Tree Representations," Computational Linguistics, vol. 24, no. 4, pp. 613-632, 1998.

[39] E. Black, F. Jelinek, J.D. Lafferty, D.M. Magerman, R.L. Mercer, and S. Roukos, "Towards History-Based Grammars: Using Richer Models for Probabilistic Parsing," Proc. DARPA Speech and Natural Language Workshop, pp. 31-37, 1992.

[40] R. Bod and R. Scha, "Data-Oriented Language Processing: An Overview," Technical Report LP-96-13, Dept. of Computational Linguistics, Univ. of Amsterdam, The Netherlands, 1996.

[41] J. Sáchez and J. Benedi, "Consistency of Stochastic Context-Free Grammars from Probabilistic Estimation Based on Growth Transformations," IEEE Trans. Pattern Analysis and Machine Intelligence, vol. 19, no. 9, pp. 1052-1055, Sept. 1997.

[42] Z. Chi and S. Geman, "Estimation of Probabilistic Context-Free Grammars," Computational Linguistics, vol. 24, no. 2, pp. 299-305, 1998.

[43] J. Chappelier and M. Rajman, "A Generalized CYK Algorithm for Parsing Stochastic CFG," Proc. Tabulation in Parsing and Deduction, pp. 133-137, Apr. 1998, ftp://ftp.inria.fr/INRIA/Projects/Atoll/ TAPD98/chappelier.ps.gz.

[44] J. Carroll, T. Briscoe, and A. Sanfilippo, "Parser Evaluation: A Survey and a New Proposal," Proc. Int'l Conf. Language Resources and Evaluation, pp. 447-454, 1998.

[45] A. Krotov, R. Gaizauskas, M. Hepple, and Y. Wilks, "Compacting the Penn Treebank Grammar," Proc. COLING-ACL'98 Joint Conf. (17th Int'l Conf. Computational Linguistics, and 36th Ann. Meeting of the Assoc. Computational Linguistics), pp. 699-703, 1998.

[46] F. Pereira and Y. Schabes, "Inside-Outside Re-Estimation from Partially Bracketed Corpora," Proc. 30th Ann. Meeting of the ACL, pp. 128-135, 1992. 


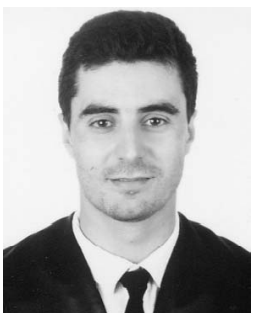

Jose L. Verdú-Mas graduated with the degee in computer science in 1995 from the Universidad Politecnica de Valencia, Spain. He is about to receive the $\mathrm{PhD}$ degree in computer science from the Universidad de Alicante, Spain. In 1995, he joined the Departamento de Lenguajes y Sistemas Informaticos at the Universidad de Alicante, teaching advanced algorithms and formal languages and automata theory. His research interests include grammar induction from stochastic samples, probabilistic automata, and finite-state methods in automatic translation.

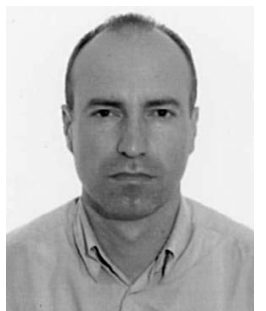

Rafael C. Carrasco received the degree in physics from the University of Valencia in 1987. $\mathrm{He}$ received the $\mathrm{PhD}$ degree in theoretical physics (University of Valencia, 1991) and the $\mathrm{PhD}$ degree in computer science (University of Alicante, 1997). In 1992, he joined the Departamento de Lenguajes y Sistemas Informaticos at the University of Alicante, where he is teaching, as a professor, formal languages and automata theory, algorithmics, and markup languages. Since 2002, he has led the technology section of the Miguel de Cervantes digital library (http://www.cervantesvirtual.com). His research interests include grammar induction from stochastic samples, probabilistic automata, recurrent neural networks and rule encoding, markup languages and digital libraries, finite-state methods in automatic translation, and computer simulation of photonuclear reactions.

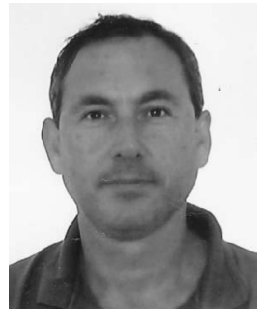

Jorge Calera-Rubio received the BSc degree in physics from the Universidad Autonoma de Madrid in 1987. He received the PhD degree in physics from the Universidad de Valencia in 1996. In 1995, he joined the Departamento de Lenguajes y Sistemas Informaticos at the Universidad de Alicante, teaching formal languages and automata theory, compilers, and machine learning. His research interests include grammatical inference, pattern recognition, natural language processing, and data compression.

$\triangleright$ For more information on this or any other computing topic, please visit our Digital Library at www.computer.org/publications/dlib. 